ARTICLE

\title{
Polychromatic solar energy conversion in pigment- protein chimeras that unite the two kingdoms of (bacterio)chlorophyll-based photosynthesis
}

\author{
Juntai Liu (D) 1 , Vincent M. Friebe $\mathbb{D}^{2}$, Raoul N. Frese $^{2} \&$ Michael R. Jones (iD) ${ }^{1 凶}$
}

Natural photosynthesis can be divided between the chlorophyll-containing plants, algae and cyanobacteria that make up the oxygenic phototrophs and a diversity of bacteriochlorophyllcontaining bacteria that make up the anoxygenic phototrophs. Photosynthetic light harvesting and reaction centre proteins from both kingdoms have been exploited for solar energy conversion, solar fuel synthesis and sensing technologies, but the energy harvesting abilities of these devices are limited by each protein's individual palette of pigments. In this work we demonstrate a range of genetically-encoded, self-assembling photosystems in which recombinant plant light harvesting complexes are covalently locked with reaction centres from a purple photosynthetic bacterium, producing macromolecular chimeras that display mechanisms of polychromatic solar energy harvesting and conversion. Our findings illustrate the power of a synthetic biology approach in which bottom-up construction of photosystems using naturally diverse but mechanistically complementary components can be achieved in a predictable fashion through the encoding of adaptable, plug-and-play covalent interfaces.

\footnotetext{
${ }^{1}$ School of Biochemistry, Faculty of Life Sciences, Biomedical Sciences Building, University of Bristol, University Walk, Bristol BS8 1TD, UK. ${ }^{2}$ Department of Physics and Astronomy, LaserLaB Amsterdam, VU University Amsterdam, De Boelelaan 1081, Amsterdam 1081 HV, The Netherlands. ${ }^{凶}$ email: m.r.jones@bristol.ac.uk
} 
O ur everyday experience of photosynthesis is dominated by the blue/red-absorbing pigment chlorophyll, a magnesium tetrapyrrole that acts as both a harvester of solar energy and a carrier of electrons and holes. Variants of this versatile molecule, principally chlorophyll $a$ and chlorophyll $b$, are found in the plants, algae and cyanobacteria that make up the oxygenic phototrophs. Less well-known are the anoxygenic phototrophs, bacteria that use electron donors other than water and have one or more variants of bacteriochlorophyll as their principal photosynthetic pigment. Although these bacteria are less obvious in our environment, oxygen-tolerant species are widespread in oceanic surface waters where they make a sizeable contribution to global solar energy conversion ${ }^{1}$. A few species, including the bacteriochlorophyll a-containing Rhodobacter (Rba.) sphaeroides, have played major roles in our understanding of excitation energy transfer in light-harvesting "antenna" complexes (LHCs) $)^{2-4}$ and charge separation in photochemical reaction centres $(\mathrm{RCs})^{5,6}$.

Improving the performance of photosynthesis and finding new ways to exploit natural solar energy conversion have become important research topics 7,8 , and there is growing interest in the use of photosynthetic proteins as environmentally benign components in biohybrid devices for solar energy conversion ${ }^{9-14}$. Photoexcitation of a RC in such a device triggers intra-protein charge separation, producing a potential difference between opposite "poles" of the protein that drives subsequent electron transfer to create a photocurrent and photovoltage. In addition to solar energy conversion per se, proposed applications of photoprotein devices have included biosensing, light/UV sensing, touch sensing and solar fuel synthesis ${ }^{9-16}$. Photosynthetic proteins are attractive as device components because they are environmentally sustainable and benign, they achieve solar energy conversion with a very high quantum efficiency (charges separated per photon absorbed) and they can be adapted to purpose through protein engineering. However, a limitation is their selective use of available solar energy ${ }^{7,8}$, a consequence of their particular palette of lightharvesting pigments (Fig. 1a). This can be evidenced in devices through the recording of action spectra of external quantum efficiency (EQE-the number of charges transferred per incident photon), which exhibit peaks and troughs that correspond to the absorbance spectra of the particular light-harvesting pigments that are coupled to charge separation in the device ${ }^{12,17-21}$.

One option for the expansion of a protein's light-harvesting capacity is to attach to it chromophores such as synthetic dyes $^{22-24}$ or emissive nanoparticles ${ }^{25-27}$. Drawbacks of this approach are that synthetic dyes are often expensive and prone to photobleaching ${ }^{26}$, while fluorescent nanoparticles can be toxic and achieving well-controlled assembly of protein-nanoparticle conjugates is challenging 28 . More akin to the present study is a report of a fusion protein between a single Yellow Fluorescent Protein (YFP) and the purple bacterial RC, which has the effect of somewhat enhancing light harvesting in a region where RC absorbance is weak by adding a single chromophore ${ }^{29}$.

A striking observation is the complementary nature of the absorbance spectra of chlorophyll and bacteriochlorophyll photosystems (Fig. 1a). This is enabled by the somewhat different electronic structures of their principal pigments (Supplementary Fig. 1a) and facilitates the occupancy of complementary ecological niches by oxygenic and anoxygenic phototrophs. Chlorophyll absorbs most strongly in the blue and red whereas the absorbance of bacteriochlorophyll is shifted to the near-ultraviolet and near-infrared. The absorbance spectra of plant and bacterial carotenoids between 400 and $600 \mathrm{~nm}$ are also somewhat complementary (Fig. 1a). Thus, anoxygenic phototrophs harvest parts of the solar spectrum which oxygenic phototrophs do not absorb well, and vice versa.
Following nature's lead, here we present the use of genetic encoding to achieve in vitro self-assembly, from diverse components (Fig. 1b, c), of photoprotein "chimeras" that display polychromatic solar energy harvesting and conversion. The components are the Rba. sphaeroides $\mathrm{RC}^{5,6}$ and the $\mathrm{LHCII}^{30-33}$ and heterodimeric $\mathrm{LHCI}^{34-38}$ proteins from Arabidopsis (A.) thaliana (Supplementary Fig. 1b-e). Highly specific and programmable self-assembly is achieved through adaptation of these components with the constituents of a two-component protein interface domain (Supplementary Fig. 1f) that covalently locks together two photosynthetic membrane proteins that have no natural propensity to associate in a specific and/or controllable manner. The resulting macromolecular, adaptable chimeric photosystems have defined compositions, and display solar energy conversion across the near-UV, visible and near-IR.

\section{Results}

Solar energy conversion by unadapted photosystem components. We first looked at whether plant LHCIIs can pass harvested energy to purple bacterial RCs in dilute solution in the absence of complementary genetic adaptations to promote specific heterodimerisation (complexes defined as "unadapted"). On receipt of excitation energy, photochemical charge separation in the Rba. sphaeroides RC is a rapid four-step process (Fig. 1d) that produces a metastable oxidised primary electron donor $\left(\mathrm{P}^{2} 70^{+}\right)$ and reduced acceptor ubiquinone $\left(\mathrm{Q}_{\mathrm{B}}{ }^{-}\right)$; energy transfer can therefore be detected as a quenching of LHC emission accompanied by an enhancement of P870 oxidation. Although bacterial RCs and plant LHCIIs (see Methods for sources) have overlapping absorbance and emission spectra between 640 and 800 nm (Fig. 1b), no appreciable energy transfer was observed when wild-type (WT) RCs were mixed in solution with an LHCII because they have no capacity for binding to one another. The addition of purified wild-type (WT) RCs did not significantly reduce emission from LHCII (Fig. 2a) and photo-oxidative bleaching of the absorbance band of this RC's P870 primary electron donor BChls in response to $650 \mathrm{~nm}$ excitation was not significantly enhanced by the addition of LHCII (Fig. 2b), which absorbs strongly at this wavelength (Fig. 1b).

In comparison to LHCII, the spectral overlap ( $J$ ) between LHC emission and RC absorbance is $\sim 80 \%$ larger in the case of LHCI (Fig. 1c, Supplementary Table 1) which contains a pair of "redform" chlorophyll $a$ that possess a charge-transfer state that mixes with the low-energy exciton state ${ }^{38}$. Although the addition of WT RCs did bring about a decrease in LHCI emission (Fig. 2a), there was no associated significant increase in RC P870 photobleaching in the presence of LHCI (Fig. 2c), leading to the conclusion that the observed emission quenching was not due to energy transfer. Protein concentrations used for the fluorescence measurements were too low ( $\max$ absorbance $<0.07$ ) for this drop in LHCI emission to be attributable to reabsorption by the added RCs, and an equivalent drop was not seen for LHCII and WT RCs at similar concentrations (Fig. 2a). As it is known that the emission quantum yield of LHCI in vitro is much more sensitive to its environment than is the case for $\mathrm{LHCII}^{36}$, the observed drop in LHCI emission on adding WT RCs is attributed to a change in its intrinsic quantum yield rather than being a signature of energy transfer.

Although no significant energy transfer was seen between these proteins in dilute solution, to establish the principle that plant LHCs can pass energy to bacterial RCs when brought sufficiently close together, mixtures of LHC and WT RC proteins were adhered to a nanostructured silver cathode and their capacity for generating photocurrents examined (see Methods). In this photoelectrochemical system (Fig. 2d) cytochrome $c$ (cyt c) is used to "wire" charge separation in the RC to the cathode, 

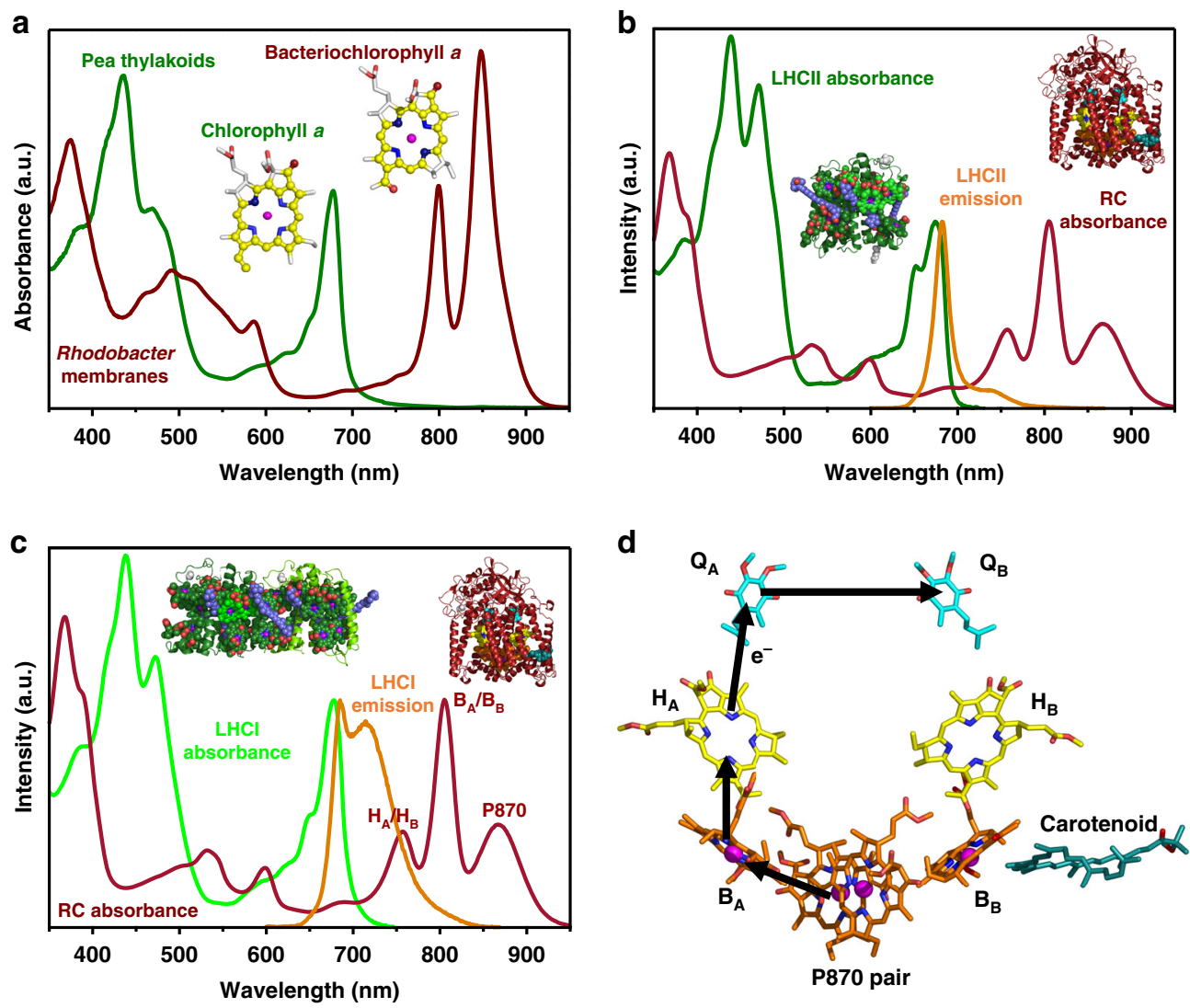

Fig. 1 Component absorbance, emission and mechanism. a Thylakoid membranes from oxygenic phototrophs such as pea and chromatophore membranes from anoxygenic phototrophs such as Rba. sphaeroides have complementary absorbance spectra due to differences in the electronic structures of the macrocycle $\pi$ electron systems of chlorophyll and bacteriochlorophyll (see also Supplementary Fig. 1). b The major plant light-harvesting complex LHCII harvests solar energy in regions where absorbance by Rba. sphaeroides RCs is weak, notably around $650 \mathrm{~nm}$, and its emission spectrum overlaps the absorbance spectrum of the RC between 640 and $800 \mathrm{~nm}$. c The red-enhanced emission spectrum of heterodimeric plant LHCI has a stronger overlap with the absorbance spectrum of the Rba. sphaeroides RC, particularly the coincident absorbance bands of the bacteriopheophytins $\left(\mathrm{H}_{A} / \mathrm{H}_{B}\right)$. d Architecture of the RC cofactors and the route of four-step charge separation which oxidises P870 and reduces $\mathrm{Q}_{\mathrm{B}}$. The bacteriochlorophylls (orange carbons) and bacteriopheophytins (yellow carbons) give rise to the absorbance bands labelled in $\mathbf{c}$. Further descriptions of pigment-protein structures and their sources are given in Supplementary Fig. 1.

and ubiquinone-0 $\left(\mathrm{Q}_{0}\right)$ shuttles electrons to the counter electrode $\mathrm{e}^{20,39,40}$. Electrodes drop-cast with purified WT RCs produced a photocurrent in response to RC-specific $870 \mathrm{~nm}$ light and a weaker current in response to $650 \mathrm{~nm}$ excitation where RC absorbance is very low (Supplementary Fig. 2a). An EQE action spectrum showed good correspondence with the RC absorbance spectrum (Fig. 2e, magenta versus black), confirming that the photocurrent was attributable to light capture by the pigments of the RC. As expected, an electrode fabricated with purified LHCII failed to show any photocurrent response during $650 \mathrm{~nm}$ excitation of the main low-energy LHCII absorbance band (Supplementary Fig. 2a).

For electrodes fabricated from mixtures of WT RCs and LHCs, in addition to the expected RC bands the EQE spectra contained a component between 620 and $700 \mathrm{~nm}$ that corresponded to the lowenergy absorbance band of LHCII or LHCI (Fig. 2e, green). A contribution from the high-energy Soret absorbance band of LHCII or LHCI was also observed in EQE spectra (Supplementary Figs. 3a, $\mathrm{b}$ and 4). This demonstrated that bacteriochlorophyll-based purple bacterial RCs can utilise chlorophyll-based plant LHCs for energy harvesting, producing charge separation and a photocurrent response, provided they are brought within Förster resonance energy transfer (FRET) distance of one another. In this case this was realised by colocalising the two proteins on the surface of a biophotoelectrode.
Design and production of components for chimeric photosystems. In an attempt to activate chlorophyll to bacteriochlorophyll energy transfer in dilute solution, RCs and LHCs were adapted using the SpyTag/SpyCatcher protein fusion system $^{41}$ as a programmable interface (see Supplementary Note 1). When mixed in solution, highly specific binding of the short SpyTag peptide to the SpyCatcher protein domain initiates autocatalysis of an isopeptide bond between the two involving aspartate and lysine residues (Supplementary Fig. 1f), producing a single, covalently locked, water-soluble protein domain ${ }^{41}$.

To adapt the RC for LHC binding an optimised version of SpyCatcher ${ }^{42}, 106$ amino acids in length (SpyCatcher $\Delta$ ), was attached to the N-terminus of the RC PufL protein either directly (dubbed "RCC") or via a four residue linker (dubbed "RC4C") (Fig. 3a, Supplementary Table 2). Adapted RC proteins were expressed in Rba. sphaeroides (see Methods). For LHCII, Lhcb apoproteins were expressed in E. coli and mature pigment-protein monomers refolded in vitro with purified pigments ${ }^{43-46}$ (see Methods). Three LHCII proteins were designed (Fig. 3b; see Supplementary Fig. 5a for protein sequences). The first, dubbed "dLHCII", lacked 12 dispensable $\mathrm{N}$-terminal amino acids that are not resolved in available X-ray crystal structures ${ }^{30-32}$ and had a His-tag at its C-terminus (see Supplementary Note 1). The remaining two had either a truncated SpyTag variant $(\operatorname{SpyTag} \Delta)$ added to the N-terminus of the truncated Lhcbl (termed 

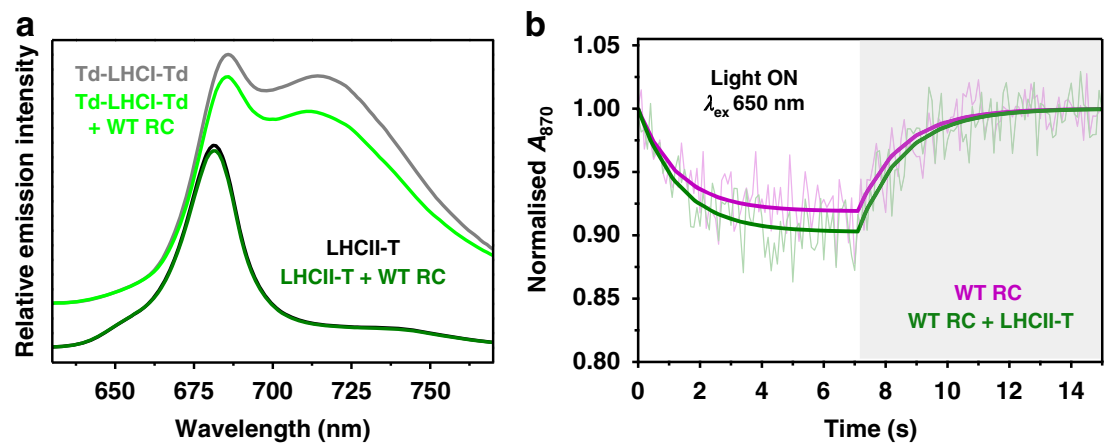

d

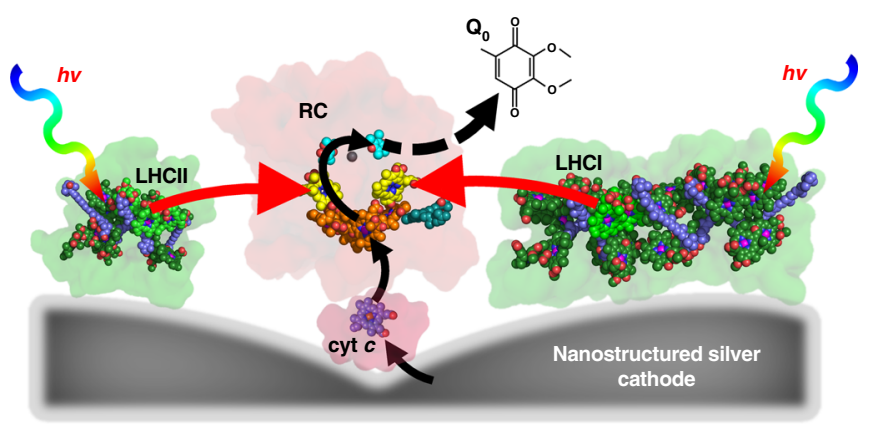

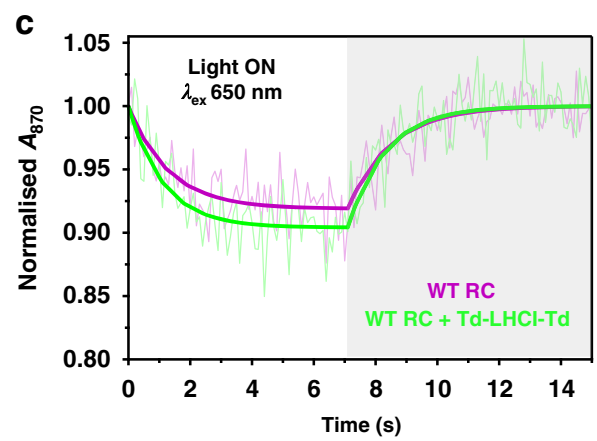

e

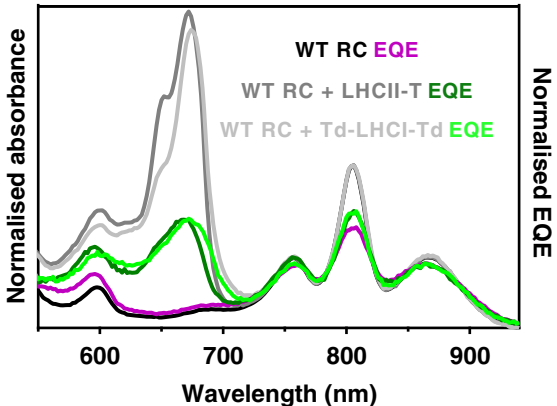

Fig. 2 Energy transfer requires co-localisation of RCs and LHCs. a LHCIl emission (excitation at $475 \mathrm{~nm}$ ) and LHCl emission (excitation at $500 \mathrm{~nm}$ ) in the absence and presence of WT RCs. The latter spectra are offset for clarity. $\mathbf{b}$ Data and fits for photobleaching and dark recovery of P870 absorbance for the WT RC in the absence and presence of LHCII (using variant LHCII-T). c Photobleaching and dark recovery of P870 absorbance in WT RCs in the absence and presence of $\mathrm{LHCl}$ (using variant Td-LHCl-Td). d Schematic of photocurrent generation on a nanostructured silver electrode; black arrows show the route of electron transfer and red arrows show energy flow. e Solution absorbance and EQE spectra for WT RCs compared with those for mixtures of WT RCs with either LHCII-T or Td-LHCI-Td. The absorbance spectra were normalised at $804 \mathrm{~nm}$, while each EQE spectrum was normalised to the corresponding absorbance spectrum at the maximum of the long-wavelength P870 band.

Td-dLHCII) or the full SpyTag sequence added to the C-terminus of the full Lhcb1 (termed LHCII-T) (Fig. 3b).

Adapted heterodimeric LHCI proteins (Fig. 3c; see Supplementary Fig. $5 \mathrm{a}$ for protein sequences) were also refolded from apoproteins expressed in E. coli ${ }^{34,38,47,48}$. This involved mixing SpyTag $\Delta$-adapted Lhca4 protein (Td-L4) with either unadapted Lhcal protein (L1) or SpyTag $\Delta$-adapted Lhcal protein (Td-L1) to produce LHCI either singly or doubly modified with SpyTag $\Delta$ (termed LHCI-Td and Td-LHCI-Td, respectively). This enabled the creation of chimeras between LHCI and either one or two RCs (see further details in Supplementary Note 1).

Self-assembly of RC-LHC chimeras. Following ultracentrifugation, purified RCs and LHCIIs could be visualised on sucrose density gradients as either a red or green band, respectively (Fig. 3d, gradients 1 and 2), and these two proteins also migrated separately in gradients loaded with a mixture with only either the SpyTag or SpyCatcher adaptations (Fig. 3d, gradients 3 and 4). In contrast, mixing any SpyCatcher $\Delta$-adapted RC with any SpyTag $(\Delta)$-adapted LHCII produced a product, dubbed a "chimera", that migrated further than either monomeric protein. The two examples shown in Fig. 3d (gradients 5 and 6) are chimeras from a RC4C/Td-dLHCII mix (dubbed "RC\#LHCII") and from a RCC/ LHCII-T mix (dubbed "LHCII\#RC"). The symbol "\#” denotes the spontaneously formed SpyCatcher/SpyTag interface domain. Chimera formation could also be detected on a native blue gel (Supplementary Fig. 6a). Sodium dodecyl sulfate-polyacrylamide gel electrophoresis (SDS-PAGE) combined with western blotting using anti-His antibodies confirmed that chimera self-assembly was due to the formation of a covalent bond between the SpyTag $(\Delta)$-adapted Lhcb1 polypeptide of LHCII and the SpyCatcher $\Delta$ adapted PufL polypeptide of the RC (Supplementary Fig. 6b,
Supplementary Note 2). The reaction half-time for chimera formation varied between 10 and 90 min depending on the particular combination of adapted RC and LHCII (detailed in Supplementary Note 3).

LHCI-RC chimeras could also be assembled by incubation of LHCI-Td or Td-LHCI-Td with a threefold excess of RCC. This again produced higher molecular weight products that could be separated from unreacted RCs on blue native gels (Fig. 3e). As designed, assembly of RCC with doubly adapted Td-LHCI-Td complexes produced higher molecular weight products than with singly adapted LHCI-Td complexes (Fig. 3e, right). Equivalent results were obtained with LHCI adapted with the full SpyTag and also with RC4C (Supplementary Fig. 7a). Analysis by SDSPAGE and western blotting showed that chimera self-assembly was due to formation of a covalent bond between the SpyCatcher $\Delta$-adapted PufL of the RC and Lhca4 of a singly SpyTag $\Delta$-adapted LHCI (to form chimera LHCI\#RC) or Lhca4 and Lhcal of a doubly SpyTag $\Delta$-adapted LHCI (to form chimera RC\#LHCI\#RC) (Supplementary Fig. 7b). Sucrose density gradient ultracentrifugation (Fig. 3f) showed that LHCI\#RC chimeras (gradient 5) were clearly larger than LHCI alone (gradients 2-4) or unadapted RCs (gradients 1,3 and 4), and RC\#LHCI\#RC chimeras (gradient 6 ) were larger again.

Covalent locking of the structure enabled purification of all LHCI-RC and LHCII-RC chimeras by a combination of nickel affinity and size-exclusion chromatography, absorbance spectroscopy being used to identify fractions containing protein oligomers with the designed molar ratio (Supplementary Fig. 8).

A change in protein morphology on chimera formation could be observed by transmission electron microscopy (TEM). Images of a mix of unadapted WT RCs and dLHCII showed a large number of monodispersed, regularly sized objects of $<10 \mathrm{~nm}$ 
a

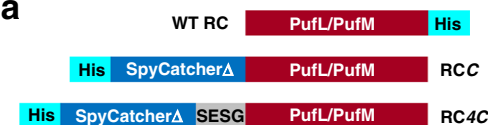

d

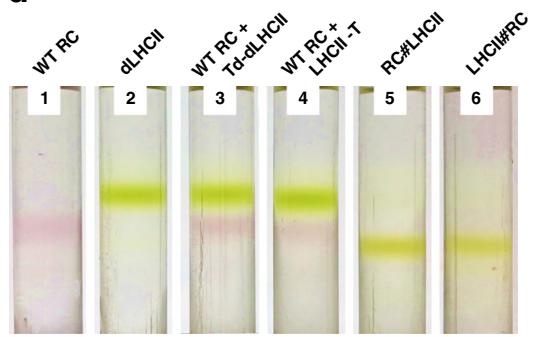

g

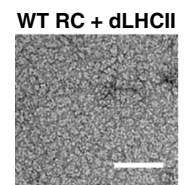

LHCI\#RC

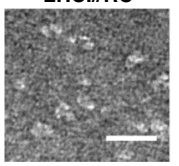

LHCII\#RC

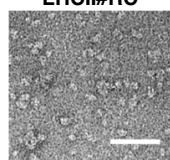

RC\#LHCI\#RC b

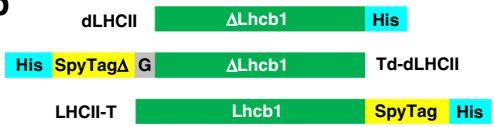

C

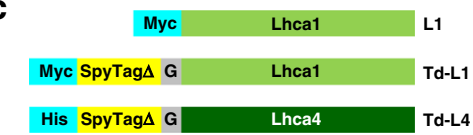

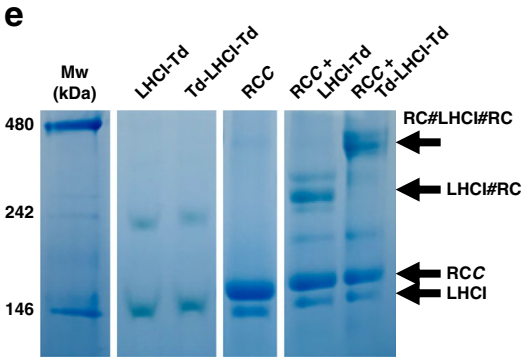

$\mathbf{h}$

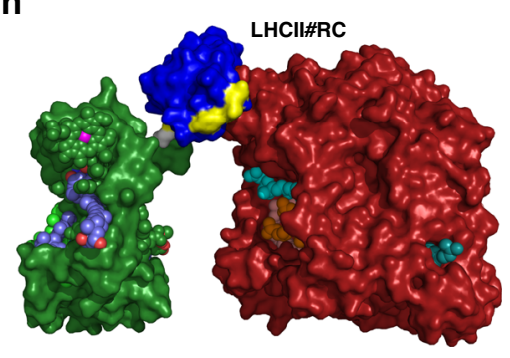

f

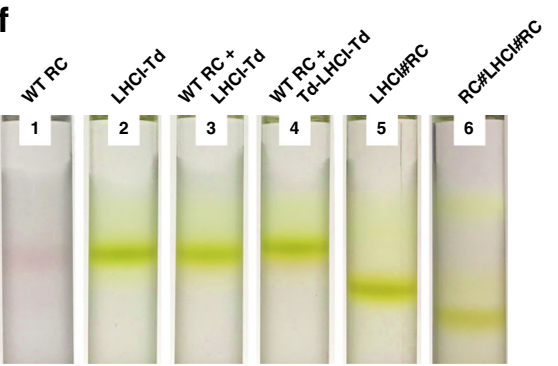

i

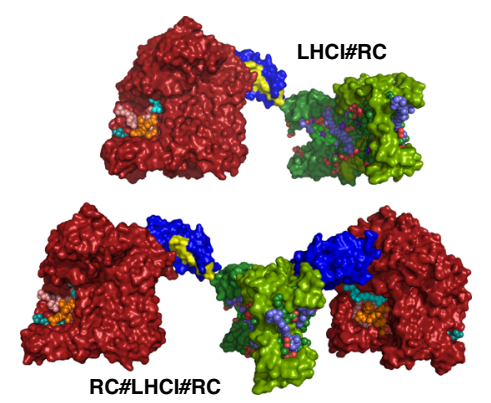

Fig. 3 Engineering and assembly of RC-LHC chimeras. a Construct designs for adaptation of the RC. For purification the WT RC was modified with a Histag on PufM. $\mathbf{b}$ Construct designs for adaptation of LHCII. The control LHCII was truncated at its N-terminus (dLHCII-see text) and was His-tagged at its C-terminus. c Construct designs for adaptation of $\mathrm{LHCl}$ which is an Lhca1/Lhca4 heterodimer. For $\mathbf{b}$ and $\mathbf{c}$ protein sequences are given in Supplementary Fig. 5a. d Sucrose density gradient fractionation of RCs (red bands) and LHClls (green bands). RC-LHCIl chimeras migrate to a lower position in gradients than either RC or LHCII monomers, with no dissociation into components. e Blue NativePAGE showing the formation of high molecular weight products by mixing LHCl-Td or Td-LHCl-Td with RCC (see Supplementary Fig. 7a for the full gel with more combinations). The multiple bands seen for the high molecular weight products are likely to be due to conformational heterogeneity. $\mathbf{f}$ Sucrose density gradient fractionation of RCs (red bands) and LHCls (green bands). LHCI\#RC chimeras and larger RC\#LHCI\#RC chimeras migrate to lower positions than either RCs or LHCl. $\mathbf{g}$ TEM images of an equimolar mixture of the WT RC and dLHCII (top/left), the LHCII\#RC chimera (top/right), the LHCI\#RC chimera (bottom/left) and the RC\#LHCI\#RC chimera (bottom/right). Additional images shown in Supplementary Fig. 17. Scale bar represents $50 \mathrm{~nm}$. h Molecular model of the LHCII\#RC chimera. The RC (maroon) N-terminally adapted with SpyCatcher $\Delta$ (blue) is covalently linked to LHCll (green) C-terminally adapted with SpyTag (yellow). Cofactor colours are as described in Supplementary Fig. 1. i Molecular models of the LHCI\#RC and RC\#LHCI\#RC chimeras. Colours as for panel $\mathbf{h}$ and Supplementary Fig. 1.

diameter (Fig. 3g, top/left), whereas images of the purified LHCII\#RC chimera revealed two-domain objects (Fig. 3g, top/ right). The purified LHCI\#RC and RC\#LHCI\#RC chimeras presented as objects with a more elongated morphology owing to the presence of one or two RCs and the heterodimeric LHCI (Fig. 3g, bottom). Molecular models of these chimeras, based on available X-ray crystal structures for the RC, LHCII, LHCI and SpyCatcher/Tag, are shown in Fig. $3 \mathrm{~h}$, i.

Chlorophyll to bacteriochlorophyll energy transfer. In solution, LHCII emission was quenched within each chimera in comparison to a control sample formed from an equivalent mix of the SpyTag-adapted LHCII and WT RCs (Fig. 4a; see spectra and other combinations in Supplementary Fig. 9). This was indicative of energy transfer, likely through a FRET mechanism at the distances implied by the chimera models (Fig. 3h, i), that was activated in these proteins in dilute solution by physically linking the RC to the LHCII. These trends, observed with $650 \mathrm{~nm}$ excitation, were also seen in data on the same complexes obtained with other three excitation wavelengths, with no variation in emission spectrum line shape (Supplementary Fig. 9). As well as being diagnostic of correctly refolded LHCII proteins, this lack of dependence of emission spectrum on excitation wavelength showed that the reduction in LHCII emission on chimera formation was not due to parasitic RC absorbance, which would be expected to be wavelength dependent (and also seen when WT RCs were mixed with each LHCII).

To determine the fate of transferred energy, measurements of RC P870 photooxidation in response to $650 \mathrm{~nm}$ excitation were carried out on the LHCII\#RC and RC\#LHCII chimeras and fitted to a simple interconversion reaction (Eq. (1); all parameters are summarised in Supplementary Table 3). Bleaching of $870 \mathrm{~nm}$ absorbance was stronger in LHCII\#RC chimeras than in controls comprising the RCC protein alone or a mixture of RCC with unadapted dLHCII complexes (Fig. 4b). The same was found for the RC\#LHCII chimera (Supplementary Fig. 10a). Hence, decreased emission by the LHCII energy donor was accompanied by enhanced photooxidation of the RC energy acceptor, confirming energy transfer between the two proteins in solution that was switched on only after linking them by the SpyCatcher/ Tag domain.

Turning to LHCI, a greater reduction of LHCI emission was seen on forming either LHCI\#RC or RC\#LHCI\#RC chimeras than after mixing the same adapted LHCI proteins with WT RCs (Fig. 4c; and other combinations are shown in Supplementary Fig. 11). This effect was again seen to be independent of excitation wavelength (Supplementary Fig. 11a) showing it was not due to the absorbance of excitation light by the tethered $\mathrm{RC}(\mathrm{s})$. This emission quenching was accompanied by significant 


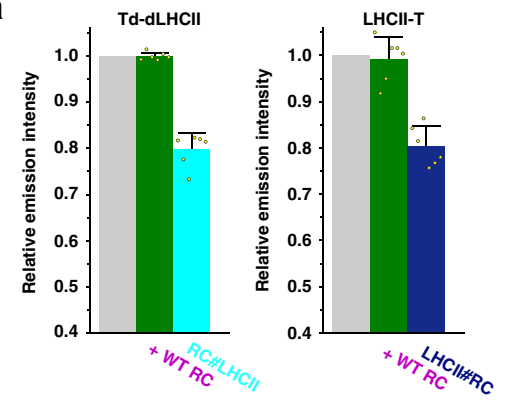

b

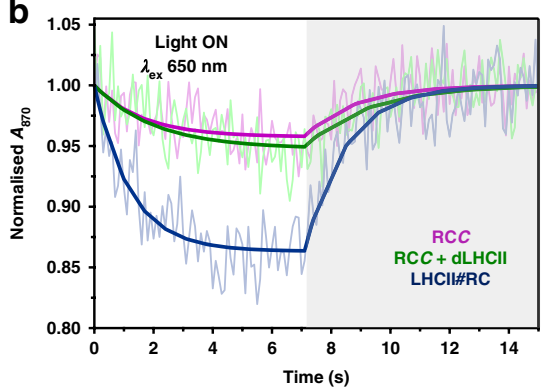

g

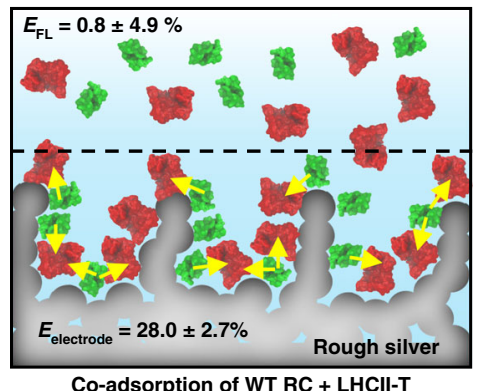

C
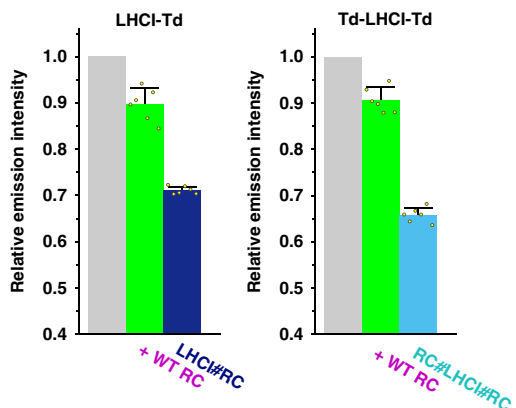

d

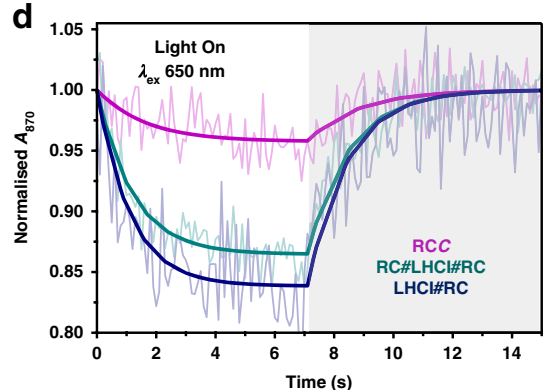

h

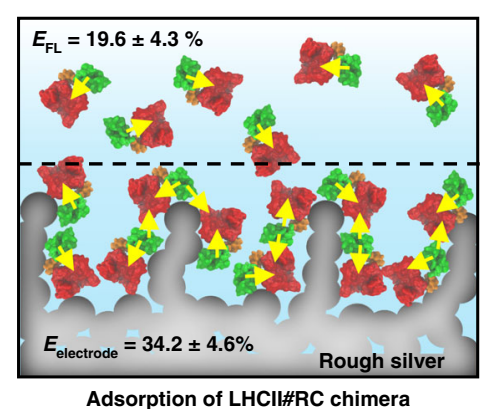

e

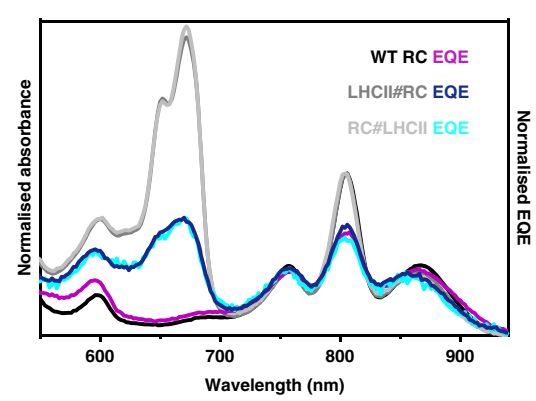

$f$

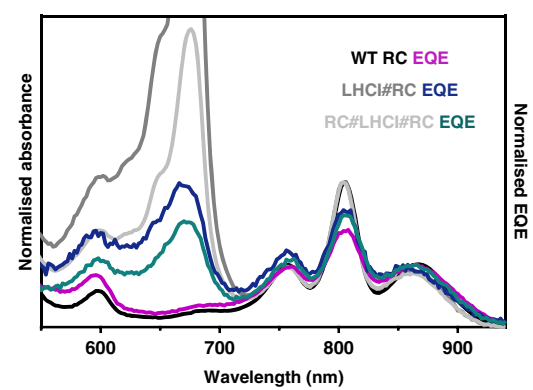

i

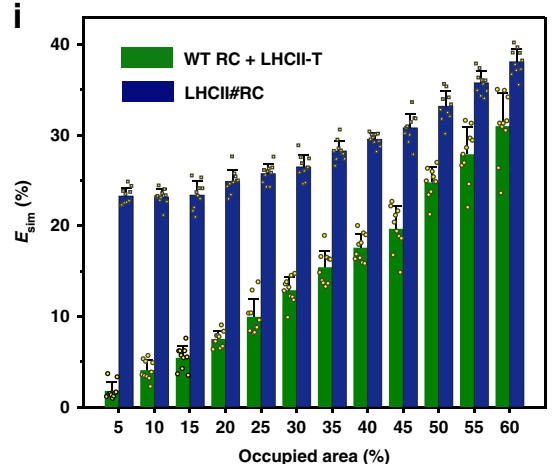

Fig. 4 Energy transfer in chimeras in solution and on surfaces. a Emission at $682 \mathrm{~nm}$ from (left) the Td-dLHCll protein alone (grey), after addition of a twofold excess of WT RCs (green) and in a LHCII\#RC chimera (cyan) formed on mixing with a twofold excess of RCC, and (right) equivalent data for LHCII-T. b Data and fits for photobleaching and dark recovery of P870 absorbance in RCC, a 1:1 RCC plus dLHCII mixture and the LHCII\#RC chimera. c Emission from (left) LHCl-Td alone (grey), after addition of a threefold excess of WT RCs (green) and in a LHCI\#RC chimera (navy) formed on mixing with a threefold excess of RCC, and (right) equivalent data for Td-LHCl-Td. Error bars in a and c represent standard deviations from six repeats (three technical repeats of two biological repeats) and data points are shown as overlaid circles. $\mathbf{d}$ Data and fits for photobleaching and dark recovery of P870 absorbance in RCC and the two RC-LHCl chimeras. e Solution absorbance and EQE spectra for WT RCs compared with those for the two LHCII-RC chimeras. $\mathbf{f}$ Solution absorbance and EQE spectra for WT RCs compared with those for the two RC-LHCl chimeras. $\mathbf{g}$ Schematic of adsorption of independent RC (red) and $\mathrm{LHCII}$ (green) complexes on an electrode. Yellow arrows indicate possible energy transfer connections. $\mathbf{h}$ Equivalent schematic of adsorption of RC\#LHCII chimeras. i Simulated apparent ET efficiencies as a function of packing density for independent RC and LHCII proteins or the LHCII\#RC chimera. Error bars represent standard deviations from ten simulation repeats. Individual data points are represented by circles or squares.

enhancement of P870 photooxidation in LHCI chimeras with one or two RCC, compared to that seen with RCC alone (Fig. 4d), confirming energy transfer. Doubly modified RC\#LHCI\#RC complexes showed less P870 bleaching than LHCI\#RC complexes due to two tethered RCs competing for the exciton reservoir rather than one (see below).

Purified chimeras were also adhered to nanostructured silver electrodes to test their functionality. All were able to generate photocurrents, showing that dynamic interactions between the $\mathrm{RC}$, cyt $c$ and ubiquinone at the electrode surface, required for the generation of a photocurrent, were not obstructed by attaching the RC to LHCII or LHCI. All EQE action spectra recorded for chimeras exhibited low-energy (Fig. 4e, f) and high-energy chlorophyll bands (Supplementary Figs. 3c, d and 4, green shading) indicating photocurrent generation powered by LHC absorbance.
Energy transfer efficiency in chimeras. Apparent efficiencies of energy transfer from LHCII or LHCI to the RC in solution were estimated either from data on emission of the LHC energy donor $\left(E_{\mathrm{FL}}\right)$ or from data on photobleaching of the RC energy acceptor $\left(E_{\mathrm{P} 870}\right)$ (see Methods, Eqs. (2)-(4)). Efficiency $E_{\mathrm{FL}}$ was based on the additional quenching of LHC emission in a chimera relative to that in a compositionally matched mixture of the relevant LHC variant and WT RCs (Eqs. (2) and (3)) or additional quenching in an $\mathrm{LHC} / \mathrm{WT}$ RC mixture relative to that in a concentrationmatched LHC-only sample. Efficiency $E_{\mathrm{P} 870}$ was based on the enhanced rate of RC $\mathrm{P} 870$ photobleaching in a chimera relative to a matched RC-only control (Eq. 4).

Values of $E_{\mathrm{P} 870}$ calculated from experimental data are shown in Table 1. The efficiency of energy transfer was low in mixtures of WT RCs with SpyTag-adapted LHCIIs or LHCIs, consistent with expectations for a dilute $(500 \mathrm{nM})$ solution of two proteins with 
Table 1 Apparent energy transfer efficiencies and associated parameters.

\begin{tabular}{|c|c|c|c|c|}
\hline System & $E_{\mathrm{P870}} \mathrm{a}^{\mathrm{a}}(\%)$ & $E_{\mathrm{FL}} \mathrm{a}^{(\%)}$ & $E_{\text {electrode }}{ }^{a}(\%)$ & $U_{\text {LHC }}(\%)$ \\
\hline $\mathrm{LHCll}-\mathrm{T}+\mathrm{WT}$ RC & $1.2 \pm 1.4^{b}$ & $0.8 \pm 4.9$ & $28.0 \pm 2.7$ & 109.1 \\
\hline LHCII\#RC & $22.8 \pm 5.1$ & $19.6 \pm 4.3$ & $34.2 \pm 4.6$ & 163.2 \\
\hline RC\#LHCII & $21.0 \pm 5.5$ & $20.3 \pm 3.6$ & $33.5 \pm 5.5$ & 172.1 \\
\hline $\mathrm{LHCl} \# \mathrm{RC}$ & $20.2 \pm 4.6$ & $20.7 \pm 0.9$ & $24.8 \pm 1.6$ & 219.9 \\
\hline WT RC + Td-LHCl-Td & $3.3 \pm 2.1^{b, c}$ & $9.3 \pm 2.8^{c}$ & $34.5 \pm 2.3$ & 130.0 \\
\hline RC\#LHCI\#RC & $29.1 \pm 6.3^{d}$ & $27.4 \pm 1.8^{d}$ & $29.0 \pm 1.3$ & 94.9 \\
\hline
\end{tabular}

no propensity to associate (see Supplementary Fig. 12 and Supplementary Table 4 for other control combinations). In marked contrast, $E_{\mathrm{P} 870}$ was over $20 \%$ in the corresponding RCLHCII or RC-LHCI chimera (Table 1). For all chimeras the value of $E_{\mathrm{FL}}$ derived from LHC emission data was in excellent agreement with the values of $E_{\mathrm{P} 870}$ derived from $\mathrm{RC}$ absorbance data (Table 1). This correspondence between independently determined efficiencies from separate data sets reinforced the conclusion that energy transfer was taking place from the plant LHCs to the bacterial RCs within the chimera.

"On electrode" apparent energy transfer efficiencies ( $\left.E_{\text {electrode }}\right)$ were also determined from the EQE action spectra, as described in Methods. In general, values of $E_{\text {electrode }}$ were higher than either estimate of energy transfer efficiency in solution (Table 1$)$. This was particularly striking for mixtures of WT RCs and $\operatorname{SpyTag}(\Delta)$ adapted LHCII or LHCI (shown schematically in Fig. $4 \mathrm{~g}$ ) where energy transfer in solution had a very low apparent efficiency. However, for the RC/LHCII chimeras in particular the value of $E_{\text {electrode }}$ was also substantially higher than $E_{\mathrm{P} 870}$ or $E_{\mathrm{FL}}$ (Table 1, Fig. $4 \mathrm{~h}$ ), suggesting that adhering the chimeras to a surface turned on inter-chimera ET that supplemented the intra-chimera ET observed in solution. This effect was less pronounced for the RC/ LHCI chimeras, particularly for complex RC\#LHCI\#RC where there were already two RCs per LHCI antenna (Table 1).

To examine whether the benefits of pre-linking RCs and LHCs in a chimera would be seen across a range of surface packing densities, a 2D Monte Carlo simulation was carried out as detailed in Supplementary Notes 4 and 5 (and summarised in Supplementary Fig. 13). In this either LHCII\#RC chimeras or a mixture of LHCII-T and WT RC proteins were represented as hard-discs on a $2 \mathrm{D}$ surface and centre-to-centre distances calculated as a function of packing density. The outcome of this simulation was an apparent energy transfer efficiency $\left(E_{\text {sim }}\right)$ based on how protein packing densities affected overall interprotein distances. In the high packing regime, $E_{\text {sim }}$ was in good agreement with the slightly higher $E_{\text {electrode }}$ determined for chimeras than for a mixture of unadapted proteins (Fig. 4i, right). As the packing density dropped to a low value (right to left in Fig. 4i), $E_{\text {sim }}$ for the chimeras gradually declined to around the values for $E_{\mathrm{P} 870}$ and $E_{\mathrm{FL}}$ estimated for the LHCII\#RC chimera in solution $(22.8 \% / 19.6 \%)$. In contrast, $E_{\text {sim }}$ for the protein mixture declined steeply to less than $2 \%$ at the lowest packing density, again in agreement with estimates of $E_{\mathrm{P} 870}$ and $E_{\mathrm{FL}}$ for the protein mixture in solution $(1.2 \% / 0.8 \%)$. This reinforced the conclusion that pre-tethering of the RC and LHCII protein into a chimera brought an added benefit even under conditions where co-localisation of the proteins on a surface switched on energy transfer between the two irrespective of tethering. Presumably pre-tethering can mitigate against situations where, for example, formation of RC-rich or
LHCII-rich sub-domains and sub-optimal mixing can lead to some proteins being outside the FRET distance (Supplementary Fig. 14, marked with blue triangles).

The EQE spectra were also used to estimate the percentage improvement in the use of visible light by an LHC/RC biophotoelectrode compared to a RC bio-photoelectrode. Consistent

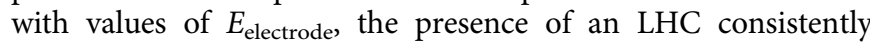
boosted the use of visible light, with the strongest effects seen for electrodes fabricated from chimeras (see Methods and Table 1).

\section{Discussion}

The data establish that it is possible to genetically encode in vitro self-assembly of a hybrid chlorophyll/bacteriochlorophyll solar energy conversion system using a highly specific split-interface domain. To our knowledge such combinations of chlorin and bacteriochlorin pigments are not used for light harvesting in nature, although in green sulfur bacteria the multiple BChl $a$ light harvesting and electron transfer cofactors of the RC are supplemented by four molecules of Chl $a$ that are used electron acceptors during charge separation ${ }^{49}$. In a similar vein, in the related heliobacterial RC the multiple $\mathrm{BChl} g$ (an isomer of Chl $a$ ) cofactors are supplemented by two molecules of $8^{1}$-hydroxychlorophyll $a$ that also act as electron transfer acceptors ${ }^{50}$. Hence some organisms have evolved to supplement bacteriochlorin cofactors with chlorins to achieve charge separation, but not to expand solar energy harvesting in the way demonstrated here.

The SpyCatcher/Tag system provided a versatile means of constructing self-assembling hybrid photosystems. LHCII could be modified with SpyTag at either its $\mathrm{N}$ - or C-terminus, and by also using heterodimeric LHCI proteins that were either singly or doubly SpyTag modified the oligomeric state of the chimeras could be varied between heterodimers (RC\#LHCII and LHCII\#RC), heterotrimers (LHCI\#RC) and heterotetramers (RC\#LHCI\#RC). The SpyCatcher/Tag linking domain produced predictable and stable products due to its very high partner specificity and the autocatalytic formation of a locking covalent bond. This binding reaction, which under the present conditions was found to have a half-time of between 10 and $90 \mathrm{~min}$, was irreversible, relatively insensitive to reaction conditions and was free from side products (i.e. a failed reaction did not lead to depletion of reactants). The assembly strategy used, using E. coli and Rba. sphaeroides as separate bacterial factories for the synthesis of protein components that could be assembled in vitro, avoided the need to re-engineer a host organism to be able to produce both chlorophyll and bacteriochlorophyll (and different types of carotenoid). This methodology therefore provides a route for the bottom-up redesign of a photosystem in vitro despite the challenges of working with large, multi-component integral membrane complexes. 


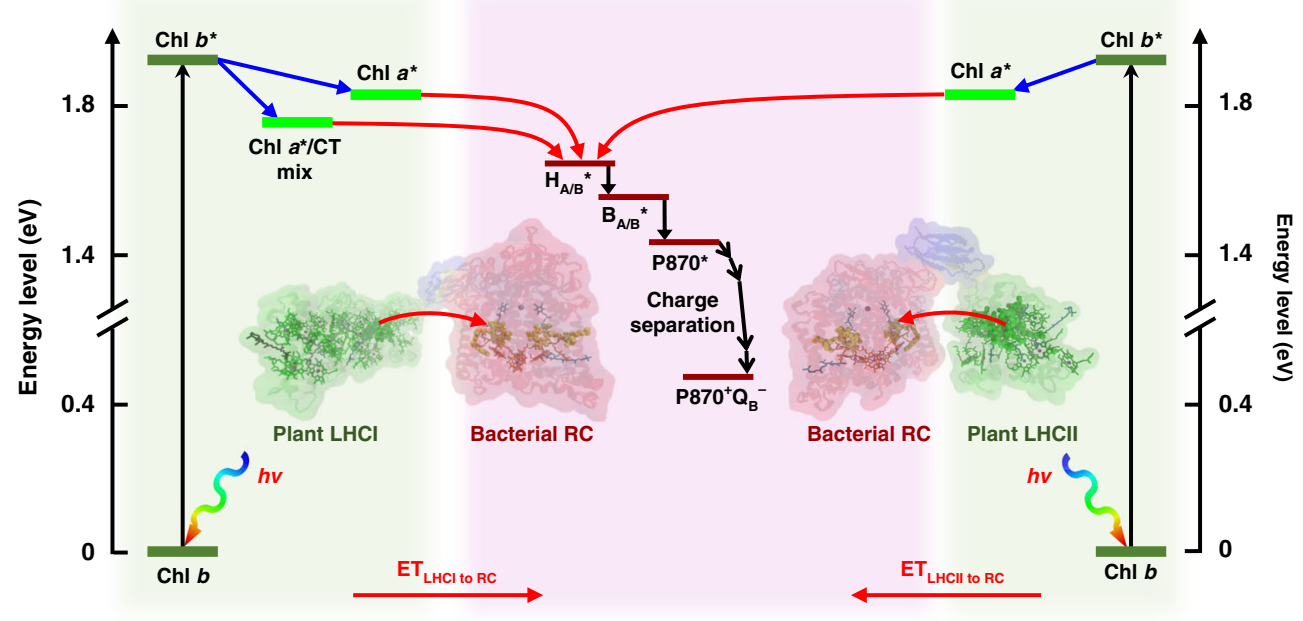

Fig. 5 Solar energy conversion in chimeras. Energy flow within $\mathrm{LHCll}$ or $\mathrm{LHCl}$ is from higher energy chlorophyll $b$ to lower energy chlorophyll $a$. LHCl also exhibits a red-shifted emissive state with mixed excitonic/charge-transfer (CT) character. Excited state energy entering the RC via the bacteriopheophytins $\left(H_{A / B}\right)$ migrates to the $P 870$ bacteriochlorophylls via the monomeric bacteriochlorophylls $\left(B_{A / B}\right)$, initiating charge separation to form $P 870+Q_{B}{ }^{-}$. Energy harvested by the carotenoid pigments of $\mathrm{LHCll}$ or $\mathrm{LHCl}$ (not shown) would transferred to the RC via their chlorophylls through fast internal relaxation 61 .

The mechanism of solar energy conversion operating in the chimeras, based on the well-understood photophysical properties of the component proteins, is summarised in Fig. 5. Energy captured by the pigment systems of LHCII or LHCI will be passed to the $\mathrm{RC}$ in a downhill manner, exciting the primary electron donor bacteriochlorophylls (P870*) and initiating charge separation to form $\mathrm{P}^{2} 70^{+} \mathrm{Q}_{\mathrm{B}}{ }^{-}$. Energy harvested by the chlorophyll $b$ (or carotenoid-not shown) pigments of either LHC is passed to the lower energy chlorophyll $a$. Inter-protein energy transfer is likely to involve a sub-set of red-shifted chlorophyll $a$ in either LHC, and entry of energy into the RC is likely to occur principally via the bacteriopheophytin cofactors $\left(\mathrm{H}_{\mathrm{A} / \mathrm{B}}\right)$ as their absorbance has the greatest spectral overlap with LHC emission (Fig. 1b, c).

As evident from comparing Fig. 1c with Fig. 1b, LHCI exhibits a red-enhanced fluorescence that produces an $\sim 80 \%$ stronger spectral overlap with RC absorbance (factor $J$ in Supplementary Table 1) compared to LHCII. Despite this, the efficiency of ET in the LHCI\#RC chimera was not significantly higher than that in either the LHCII\#RC or RC\#LHCII chimera. This is likely due to the reconstituted LHCI heterodimers being in a partially quenched state ${ }^{48,51}$ that reportedly reduces their quantum yield to only $29 \%$ of that of $\mathrm{LHCII}^{36}$, so counteracting the potential benefits of an enhanced spectral overlap. In agreement with this our estimates of quantum yield were $30 \%$ for LHCI-Td and $28 \%$ for TdLHCI-Td (Supplementary Table 1). In future work it might be possible to partially overcome this through SpyTag modification of LHCI in a native organism, as the quantum yield of purified native LHCI has been reported to be $\sim 64 \%$ that of LHCII, more than double that of recombinant $\mathrm{LHCI}^{36}$.

Estimates of ET efficiency in RC\#LHCI\#RC chimeras in solution were consistently higher than those for the LHCI\#RC chimera (parameters $E_{\mathrm{P} 870}$ and $E_{\mathrm{FL}}$ in Table 1), consistent with the presence of two ET acceptors in the former. Estimates of the ET efficiency to the second RC added to Lhcal in RC\#LHCI\#RC, made using Eq. (5), yielded values that were either $50 \%$ or $69 \%$ of that for transfer to the first RC attached to Lhca4. This is consistent with the presence of a relatively low-energy red-form chlorophyll $a$ dimer in the Lhca4 subunit (Supplementary Fig. 1d) that is responsible for the red-enhancement of the LHCI emission spectrum $^{36,38,47,48}$, and which may have produced more efficient ET to the RC attached to Lhca4 than that attached to Lhcal.
To conclude, this work shows that genetically adapting two diverse photosynthetic membrane proteins with the components of an extramembrane interface domain enables in vitro selfassembly of a chimeric photosystem in which UV/near-IR solar energy conversion by a bacteriochlorophyll-based RC is augmented by visible light capture by chlorophyll-based LHCs. This approach inspired by a concept of synthetic biology, to adapt naturally incompatible biological modules to interface in a standardised way through genetic encoding, creates covalently stabilised macromolecular photosystems that are predictable and programmable. In addition to providing photosynthetic structures and energy transfer pathways to explore, these polychromatic photosystems constitute interesting materials for biohybrid devices that in recent years have expanded in application beyond photoelectrochemical solar energy conversion to fuel molecule synthesis, energy storage, biosensing, touch sensing and photodetection. Finally, the demonstrated flexibility with which RCs and LHCs could be interfaced opens the possibility of constructing more elaborate, self-assembling chimeric photosystems that employ multiple orthogonal linking modules ${ }^{52,53}$ and a wider range of photosynthetic and redox proteins that, despite being separated by billions of years of evolution, can be adapted for future solar energy conversion through genetic programming.

\section{Methods}

RC expression and purification. Details of the designs of adapted RCs are described in Supplementary Note 1. All adapted RCs were expressed in a strain of Rba. sphaeroides engineered to lack light-harvesting complexes ${ }^{54,55}$. Bacterial cells grown under dark/semiaerobic conditions and harvested by centrifugation were suspended in $20 \mathrm{mM}$ tris(hydroxymethyl)aminomethane (Tris, $\mathrm{pH} 8.0$ ), supplemented with protease inhibitor and DNAase, and lysed in a cell disruptor (Constant Systems) at 20,000 psi. Cell debris was removed by centrifugation at 18,000 rpm for $15 \mathrm{~min}$ at $4{ }^{\circ} \mathrm{C}$. The supernatant was then incubated in the dark at $4{ }^{\circ} \mathrm{C}$ for $1 \mathrm{~h}$ with $1.5 \%$ (v/v) lauryldimethylamine $N$-oxide (LDAO) and $200 \mathrm{mM} \mathrm{NaCl}$. After ultracentrifugation at $38,000 \mathrm{rpm}$ for $30 \mathrm{~min}$ at $4{ }^{\circ} \mathrm{C}$ the supernatant was collected and initial purification carried out using a Ni-NTA (nitrilotriacetic acid) column (GE Healthcare). The protein eluate was concentrated and then further purified using a Superdex 200 gel filtration column (GE Healthcare) in $20 \mathrm{mM}$ Tris ( $\mathrm{pH} 8$ ) containing $0.04 \% N$-dodecyl- $\beta$-D-maltopyranoside (Tris/DDM buffer). Eluted fractions with a low 280 to $804 \mathrm{~nm}$ absorbance ratio $(<1.5)$ were pooled, concentrated and stored at $-80^{\circ} \mathrm{C}$.

LHCII expression, refolding and purification. Details of the designs of the adapted LHCII proteins are described in Supplementary Note 1. The starting point for production of the designed LHCII holoproteins was a pET-28a expression 
vector containing a modified gene encoding the Lhcb1.3 protein from A. thaliana (UniProtKB entry P04778). Modification of this gene was carried out by Gibson assembly using oligonucleotides sourced from Eurofins or using the Q5 ${ }^{\circ}$ SiteDirected Mutagenesis Kit from NEB.

Designed apoproteins were expressed in E. coli Rosetta 2 (Novagen). Protein expression was induced using $1 \mathrm{mM}$ isopropyl $\beta$-D-1-thiogalactopyranoside (IPTG) after the cell density reached $\mathrm{OD}_{600}=0.8$ and cells were then incubated at $37^{\circ} \mathrm{C}$ and 250 r.p.m. agitation for 4-8 h. Harvested cells were resuspended in Lysis Buffer $(50 \mathrm{mM}$ Tris $(\mathrm{pH} 8.0) / 2.5 \%(\mathrm{w} / \mathrm{v})$ sucrose $/ 1 \mathrm{mM}$ ethylenediaminetetraacetic acid (EDTA)) with protease inhibitor and DNase ${ }^{43}$. Resuspended cells were lysed in a cell disruptor (Constant Systems) at 25,000 psi. Separation of inclusion bodies (IBs) from other cell fractions was accomplished by centrifugation of the cell lysate at $12,000 \mathrm{~g}$ for $15 \mathrm{~min}$ at $4{ }^{\circ} \mathrm{C}$. IBs from the pellet were washed once with Detergent Buffer (20 mM Tris (pH 7.8)/200 mM NaCl/2 mM EDTA/1\% sodium deoxycholate/1\% octyl $\beta$-D-glucopyranoside (OG)/10 $\mathrm{mM}$ dithiothreitol (DTT)), three times with Triton Buffer (20 mM Tris ( $\mathrm{pH} 8.0) / 1 \mathrm{mM}$ DTT/0.5\% (w/v) Triton $\mathrm{X}-100)$ and finally with TE Buffer (20 mM Tris (pH 8.0)/1 mM EDTA). Washed IBs were stored at $-80^{\circ} \mathrm{C}$ as pellets and the protein purity was checked by SDSPAGE using quick Coomassie staining (Generon). The concentration of total protein was measured by a DC ${ }^{\mathrm{m}}$ protein assay (Bio-Rad).

Total chlorophylls and carotenoids were extracted from fresh spinach leaves ${ }^{43}$. Leaves were homogenised in cold grinding buffer $(0.4 \mathrm{M}$ sorbitol/10 mM NaCl$/ 5$ $\mathrm{mM} \mathrm{MgCl}_{2} / 0.5 \%(\mathrm{w} / \mathrm{v}$ ) milk powder/0.1 M Tricine ( $\mathrm{pH} 7.8)$ ) using a Waring immersion blender ( $20 \mathrm{~g}$ leaves per $100 \mathrm{~mL}$ buffer). Chloroplasts were separated by filtration through a nylon cloth and centrifugation of the filtrate at $1500 \mathrm{~g}$ for 10 min at $4{ }^{\circ} \mathrm{C}$. The chloroplast pellet was washed by twice resuspending in cold wash buffer ( $50 \mathrm{mM}$ sorbitol/10 mM EDTA/5 mM Tricine ( $\mathrm{pH} 7.8)$ ) and centrifuging at $10,000 \mathrm{~g}$ for $10 \mathrm{~min}$ at $4{ }^{\circ} \mathrm{C}$. Having removed all supernatant, total pigment was extracted in the dark by adding $80 \%$ acetone buffered with sodium carbonate to the chloroplasts and incubating on ice. Unextracted material was removed by centrifugation at $12,000 \mathrm{~g}$ for $15 \mathrm{~min}$ at $4{ }^{\circ} \mathrm{C}$. The supernatant was well mixed with 0.4 volumes of diethyl ether in a separating funnel. After layer separation was obtained by mixing in $0.33 \mathrm{M} \mathrm{NaCl}$, the upper, pigmented ether phase was decanted, dried by the addition of anhydrous sodium sulfate and solvent was evaporated under vacuum.

Carotenoids were extracted from washed chloroplasts ${ }^{43}$. The chloroplast pellet was resuspended in $96 \%$ ethanol buffered with sodium carbonate and incubated on ice as for the total pigment extraction except that $80 \% \mathrm{KOH}(\mathrm{w} / \mathrm{v})$ was added and the mixture incubated at $4{ }^{\circ} \mathrm{C}$ overnight. After saponification the solution was placed in a separating funnel and mixed gently with an equal volume of diethyl ether and then with 0.8 volumes of $0.33 \mathrm{M} \mathrm{NaCl}$. After distinct layers had formed the lower green phase was removed. Three volumes of water were then added with gentle mixing to clean the upper orange layer. After two layers had formed the top phase was isolated, dried with anhydrous sodium sulfate and the solvent was evaporated under vacuum.

Extracted pigments were re-dissolved in $100 \%$ acetone and their concentration was determined by absorbance spectroscopy in $80 \%$ buffered acetone $(20 \mathrm{mM}$ Tris, $\mathrm{pH}$ 8.0). Concentrations of $\mathrm{Chl} a, \mathrm{Chl} b$ and total carotenoid were calculated based on a published equation ${ }^{56}$

For refolding ${ }^{43}$, apoprotein in IBs was solubilised by resuspending in TE buffer (50 mM Tris (pH 8)/1 mM EDTA) and briefly heating to $95^{\circ} \mathrm{C}$ after mixing $1: 1$ in $2 \times$ Reconstitution Buffer (4\% (w/v) lithium dodecyl sulfate/20 mM DTT/2 mM benzamidine/10 $\mathrm{mM}$ aminocaproic acid/5\% (w/v) sucrose/200 $\mathrm{mM} 4$ - (2hydroxyethyl)-1-piperazineethanesulfonic acid (HEPES, pH 8). Purified chlorophyll $a / b$ and carotenoid pigments were pre-dissolved in ethanol and added to the apoprotein with gentle vortexing. Light was avoided after this step to prevent photodamage. The volume of ethanol was kept at no more than $4 \%$ of total reaction volume and a mass ratio of apoprotein/total pigment/carotenoid (w/w/w) equal to $40 / 25 / 4$ was used. OG was then added to $2 \%$ (w/v) and the mixture incubated on ice for $10 \mathrm{~min}$. $\mathrm{KCl}$ was then added to $200 \mathrm{mM}$ for $20 \mathrm{~min}$ on ice to precipitate LDS. The solution containing reconstituted LHCIIs was recovered after centrifugation for $10 \mathrm{~min}$ at $16,000 \mathrm{~g}$ and $4{ }^{\circ} \mathrm{C}$.

Each refolded LHCII was purified by nickel affinity chromatography ${ }^{43}$ and then by gel filtration chromatography. Fractions with the lowest $A_{470}$ to $A_{674}$ ratio and invariant emission profiles in response to 440,475 and $500 \mathrm{~nm}$ excitation were kept and pooled. Purified proteins were stored at $-80^{\circ} \mathrm{C}$ before use as concentrated solutions in Tris/DDM. An extinction coefficient at the chlorophyll $a \mathrm{Q}_{\mathrm{y}}$ band of $546,000 \mathrm{M}^{-1} \mathrm{~cm}^{-1}$ was used to estimate LHCII concentration ${ }^{25}$. The refolded LHCII complexes had absorbance spectra that were similar to one another (Supplementary Fig. 5b) and to spectra previously published by others ${ }^{43-46}$. Their emission spectra were highly similar (Supplementary Fig. 5c), and the line shapes of these spectra were invariant with excitation wavelength (Supplementary Fig. 5d), a feature diagnostic of a structurally intact LHCII. Pigment compositions were similar to those typically reported for recombinant LHCII (Supplementary Fig. 5e) ${ }^{46}$

LHCl expression, refolding and purification. Details of the designs of the adapted LHCI proteins are described in Supplementary Note 1. Expression plasmids were pET-28a containing synthetic genes sourced from Eurofins. Following apoprotein expression in E. coli, LHCI heterodimers were assembled by refolding with purified pigments as described above for $\mathrm{LHCII}^{34,36,38,47,48}$. A $20 \%$ excess (by mass) of either L1 or Td-L1 was mixed with Td-L4 to reduce the level of free Td-L4 monomer after refolding. The apoprotein:total pigment ratio was kept the same as for LHCII refolding. Nickel affinity chromatography was used to separate the Histagged LHCI dimer from residual Lhcal monomer (which was not His-tagged). Each LHCI was then further purified by gel filtration chromatography and stored at $-80^{\circ} \mathrm{C}$ before use as a concentrated solution in Tris/DDM. An extinction coefficient for the chlorophyll $a \mathrm{Q}_{\mathrm{y}}$ band equal to $1,092,000 \mathrm{M}^{-1} \mathrm{~cm}^{-1}$ was used to evaluate LHCI concentration since its chlorophyll $a$ content is approximately twice that of a refolded LHCII monomer ${ }^{34}$.

Chimera formation and verification. The standard approach to chimera formation was to mix RCs with a twofold molar excess of LHCII, or a threefold molar excess of LHCI, and then separate the chimera from unreacted components by gel filtration chromatography, using absorbance spectroscopy and each constituent molar extinction coefficient to assess the RC:LHC molar ratio in each column fraction (see Supplementary Fig. 8).

Formation of chimeras was initially verified by sucrose density gradient ultracentrifugation (Fig. 3d, f). Linear sucrose gradients were prepared by freezing and thawing $10 \mathrm{~mL}$ of $21 \%$ (w/v) sucrose in $20 \mathrm{mM}$ Tris/0.04\% DDM (pH 8.0). Each gradient was loaded with $400 \mu \mathrm{L}$ of sample with each photoprotein at a concentration of $2.5 \mu \mathrm{M}$ and then capped with $1 \mathrm{~mL}$ of $20 \mathrm{mM}$ Tris/0.04\% DDM ( $\mathrm{pH} 8.0$ ). Gradients were ultracentrifuged in a Sorvall TH-641 swing-out rotor at $38,000 \mathrm{rpm}$ for $18 \mathrm{~h}$ at $4^{\circ} \mathrm{C}$.

For native blue PAGE, precast NativePAGE 4-20\% gels (Thermo) were run in a Bis-Tris buffer system. Coomassie blue dye at $0.02 \%(\mathrm{w} / \mathrm{v})$ was used in the cathode buffer but not in the loading buffer. The gel cassette was placed in an ice bath and run at $150 \mathrm{~V}$ for $1 \mathrm{~h}$ followed by $250 \mathrm{~V}$ for $2 \mathrm{~h}^{57}$. SDS-PAGE was carried out using precast $4-20 \%$ gradient gels (Bio-Rad). A standard loading of 20 pmol RC was used. Loaded gels were run at $200 \mathrm{~V}$ for $45 \mathrm{~min}$ and stained overnight at room temperature with Quick Coomassie Stain (Generon).

Western blotting was carried out following protein transfer onto a nitrocellulose membrane (GE Healthcare) on a TE 77 PWR Semi-Dry Transfer Unit (45 mA/gel and $30 \mathrm{~min}$ with a NOVA Blot kit) in $30 \mathrm{~min}$. The membrane was blocked overnight with 5\% milk PBS-Tween (PBS/T) buffer and then incubated with horse radish peroxidase (HRP)-conjugated antibodies in the same buffer for $1 \mathrm{~h}$. The membrane was developed using $1 \times \operatorname{LumniGLO}(\mathrm{R})\left(\mathrm{CST}^{\oplus}\right)$ after rinsing the membrane three times with PBS/T buffer. Finally, the result was recorded on an ODYSSEY imaging system (LI-COR Biosciences). Re-probing of the membrane was accomplished by stripping and a repeat process of incubation in $5 \%$ milk PBS/ $\mathrm{T}$ buffer. Stripping of membrane was achieved by incubating twice in mild stripping buffer (200 mM glycine, 0.1\% SDS (w/v), 1\% Tween $20(\mathrm{v} / \mathrm{v}), \mathrm{pH} 2.2)$ for $5 \mathrm{~min}$ and twice in TBST buffer $(50 \mathrm{mM}$ Tris, $150 \mathrm{mM} \mathrm{NaCl}, 0.1 \%$ Tween $20(\mathrm{v} / \mathrm{v})$, $\mathrm{pH}$ 7.6), before finally transferring in $\mathrm{PBS} / \mathrm{T}$. For polyhistidine tag detection, a 1000-fold dilution of HRP-conjugated anti-His antibody was used (A7058, Sigma) and a 2000-fold dilution of HRP-conjugated c-Myc antibody was used for Myc tag detection (GT0002, Insight Biotech).

\section{Spectroscopy. Absorbance spectra were recorded on a Varian} Cary60 spectrophotometer and emission spectra on a Varian Cary Eclipse Fluorimeter in nitrogen-gassed, freshly prepared Tris/DDM.

Photooxidation of the RC P870 primary electron donor was measured using an optical fibre attachment for the Cary60 and a four-way cuvette holder (Ocean Optics, Inc.). For excitation, light from an HL-2000 light source (Ocean Optics, Inc.) was passed through an optical fibre and a 25-nm band-pass filter centred at $650 \mathrm{~nm}$ (Edmund Optics Ltd). Incident light intensity was approximately $0.1 \mathrm{~mW}$ $\mathrm{cm}^{-2}$, which excited $\sim 15 \%$ of the RC population. Light-on/off was controlled using the electronic shutter on the light source triggered by a TGP110 pulse generator (Aim-TTi Ltd, UK). After incubation with a 5-fold excess of ubiquinone-0 (UQ $\left.{ }_{0}\right)$ in the dark for $10 \mathrm{~min}$, samples at an RC concentration of $0.5 \mu \mathrm{M}(0.25 \mu \mathrm{M}$ with LHCI-Td) were housed in a $3 \mathrm{~mm}$ path length, four-sided microcuvette (110-15QS, Hellma Analytics). Each measurement was repeated five times and the traces were fitted to a model assuming a simple interconversion between the ground and photo-oxidised state:

$$
\mathrm{P} 870 \underset{k_{\mathrm{r}}}{\stackrel{k_{\mathrm{f}}}{\longrightarrow}} \mathrm{P} 870^{+}
$$

Parameters $k_{\mathrm{f}}$ and $k_{\mathrm{r}}$ from these fits are shown in Supplementary Table 2. All control samples had equimolar LHC and RC except a WT RC/Td-LHCI-Td mix where the molar ratio of RC to $\mathrm{LHC}$ was two.

LHC quantum yield estimation. The quantum yield of each LHC was determined by comparison to the dye DCM (4-(dicyanomethylene)-2-methyl-6-(4-dimethylaminostyryl)-4H-pyran; Sigma) dissolved in methanol. To avoid self-shading the absorbance of each LHC and DCM was set around 0.07 across the relevant spectral region (Supplementary Fig. 15a). Emission from DCM and each LHC (average of 10 measurements; Supplementary Fig. 15b) was corrected for spectral response and used to calculate their relative integral photon fluxes ${ }^{58,59}$. The value for $\Phi_{\mathrm{D}}$ was estimated with reference to $\Phi_{\mathrm{DCM}}=0.435$ (ref. ${ }^{60}$ ) and the refractive indices of water $\left(n_{\text {water }}=1.333\right)$ and methanol $\left(n_{\text {methanol }}=1.328\right)$ 
Photochronoamperometry and EQE action spectra. Nanostructured silver electrodes were prepared by subjecting thoroughly polished planar disc polycrystalline $\mathrm{Ag}$ electrodes (CHI Instruments) to an electrochemical procedure consisting of four oxidation/reduction cycles $(+0.4 \mathrm{~V}$ for $30 \mathrm{~s} /-0.4 \mathrm{~V}$ for $30 \mathrm{~s})$ in $100 \mathrm{mM} \mathrm{KCl}{ }^{20}$. Pigment-proteins at concentrations between 20 and $100 \mu \mathrm{M}$ were drop-casted onto prepared electrodes in the dark at $4{ }^{\circ} \mathrm{C}$ for $1 \mathrm{~h}$ and unbound protein was removed by repeated mechanically controlled dipping in $20 \mathrm{mM}$ Tris (pH 8) at $4{ }^{\circ} \mathrm{C}$. Coated electrodes were immersed in $20 \mathrm{mM}$ Tris (pH 8)/50 $\mu \mathrm{M}$ $\mathrm{KCl} / 20 \mu \mathrm{M}$ horse heart cyt $c / 1.5 \mathrm{mM}$ ubiquinone- $0\left(\mathrm{Q}_{0}\right)$ in a room temperature electrochemical cell fitted with an $\mathrm{Ag} / \mathrm{AgCl} / 3 \mathrm{M} \mathrm{KCl}$ reference electrode and a platinum counter electrode. Photocurrents were measured at a bias potential of $-50 \mathrm{mV}$ vs $\mathrm{Ag} / \mathrm{AgCl}$, controlled by a PGSTAT128N potentiostat (Metrohm Autolab). Illumination was supplied by 870 or $650 \mathrm{~nm}$ LED (Roithner Lasertechnik) with irradiances of 32 or $6.7 \mathrm{~mW} \mathrm{~cm}^{-2}$, respectively, at the electrode surface with about $50 \mathrm{~nm}$ FWHM (full-width at half-maximum) for both. EQE action spectra were recorded using a tungsten-halogen source passed through a monochromator (Supplementary Fig. 16a) ${ }^{20}$. All control samples had equimolar LHC and RC except a WT RC/Td-LHCI-Td mix where the molar ratio of RC to LHC was two.

Transmission electron microscopy. Negative stain TEM was carried out on an equimolar mixture of $500 \mathrm{nM}$ WT RCs and dLHCII, $500 \mathrm{nM}$ LHCII\#RC hetero-

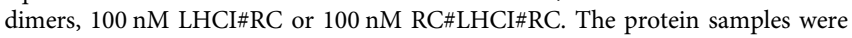
kept on ice before adsorbing to a glowing discharge-treated carbon-coated copper grid. After incubating with the grid for 1 min, excess liquid was removed with a clean filter paper. Samples were stained with 3\% uranyl acid (UA) and dried completely before imaged with a FEI Tecnai $12120 \mathrm{kV}$ BioTwin Spirit TEM system $^{27}$.

Estimation of energy transfer efficiency. Apparent efficiencies of ET were calculated from LHC emission spectra $\left(E_{\mathrm{FL}}\right)$ using

$$
E_{\mathrm{FL}}=1-\frac{\mathrm{FL}_{\text {chimera }}}{\mathrm{FL}_{\mathrm{WTRC}+\mathrm{LHC}}},
$$

where $\mathrm{FL}_{\text {chimera }}$ was the intensity of $\mathrm{LHC}$ emission in a chimera and $\mathrm{FL}_{\mathrm{WTRC}+\mathrm{LHC}}$ was that in a concentration-matched mixture of the appropriate LHCII or LHCI variant and the WT RC. A similar approach was used for estimating the apparent ET efficiency in mixtures of WT RCs and LHCII or LHCI, expressing F $\mathrm{L}_{\mathrm{WTRC}+\mathrm{LHC}}$ as a function of the emission from the same concentration of the LHC ( $\mathrm{FL}_{\mathrm{LHC}}$ ). For LHCII, where the line shape of the emission spectrum did not vary as it is a single quantum system ${ }^{61}$, maximum emission values were used in Eq. (2) as a simple measure of emission intensity. For LHCI, which has multiple distinct emission states $^{38}$, values of emission intensity $\left(\mathrm{FL}_{\text {int }}\right.$ ) were produced by integration across the emission spectrum using Eq. (3), and then applied in Eq. (2).

$$
\mathrm{FL}_{\text {int }}=\int \frac{\lambda}{h c} \mathrm{FL}_{\mathrm{LHCI}}(\lambda) \mathrm{d} \lambda .
$$

Apparent efficiencies of ET were also calculated from the rate of P870 photobleaching $\left(k_{\mathrm{f}}\right)$ from the kinetic analyses summarised in Supplementary Table 2. To enable this the intensity of the $650 \mathrm{~nm}$ excitation light used in these experiments was kept low such that no more than $\sim 15 \%$ of P870 was oxidised within the lifetime of $\mathrm{P} 80^{+}(\sim 1 \mathrm{~s})$, ensuring that photooxidation directly represented the quantity of energy received by either direct absorption by the RC or ET from the tethered LHC. The apparent efficiency of ET $\left(E_{\mathrm{P} 870}\right)$ was estimated from the rate of P870 photobleaching using

$$
E_{\mathrm{P} 870}=\frac{k_{\mathrm{f}}(\text { chimera })-k_{\mathrm{f}}(\mathrm{RC})}{k_{\mathrm{f}}(\mathrm{RC})} \frac{\int P(\lambda)\left(1-10^{-\mathrm{Abs}_{\mathrm{RC}}(\lambda)}\right) \mathrm{d} \lambda}{\int P(\lambda)\left(1-10^{-\mathrm{Abs}_{\mathrm{LHC}}(\lambda)}\right) \mathrm{d} \lambda},
$$

where $k_{\mathrm{f}}$ was the rate of P870 oxidation in a chimera or the equivalent RC-only control (RC) (Supplementary Table 3). Integration of incident photon flux $(P)$ and the 1-transmittance of RCs or LHCs as a function of wavelength provided the number of photons absorbed by either RCs or LHCIIs per unit area per second (Supplementary Table 3).

Equation (4) was also used for estimation of $E_{\text {electrode, }}$ with parameter $k_{\mathrm{f}}$ replaced by the maximum $\mathrm{EQE}$ around $650 \mathrm{~nm}$ (i.e. the same illumination region as used for measurements of P870 oxidation). The efficiency was determined by comparing the LHC's contribution to the EQE with the sample absorbance at the corresponding wavelength (Supplementary Fig. 4). In addition to enabling direct comparison of $E_{\text {electrode }}$ and $E_{\mathrm{P} 870}$, the data at $650 \mathrm{~nm}$ were not affected by parasitic absorption or unwanted emission from cyt $c, \mathrm{UQ}_{0}$ or nanostructured silver used in photocurrent measurements $\mathrm{s}^{20,40}$.

For RC\#LHCI\#RC chimeras there were two acceptors per LHCI, one connected to the Lhca1 subunit and one to the Lhca4 subunit. Efficiencies of energy transfer to the Lhcal-connected RC $\left(E_{\mathrm{a} 1}\right)$ were estimated from:

$$
E_{\mathrm{FL}, \mathrm{P} 870}=\frac{E_{\mathrm{a} 1}+E_{\mathrm{a} 4}-2 E_{\mathrm{aa}} E_{\mathrm{a} 4}}{1-E_{\mathrm{a} 1} E_{\mathrm{a} 4}},
$$

where $E_{\mathrm{FL}, \mathrm{P} 870}$ was the apparent energy transfer efficiency for the RC\#LHCI\#RC chimera estimated from either LHC fluorescence or P870 photobleaching and
$E_{\mathrm{a} 4}$ was the corresponding apparent energy transfer efficiency for the LHCI\#RC chimera where the single RC is attached to Lhca4. From $E_{\mathrm{FL}}$ the value of $E_{\mathrm{a} 1}$ was $10.4 \%$ (compared to $E_{\mathrm{a} 4}=20.7 \%$ ) and from $E_{\mathrm{P} 870}$ the value of $E_{\mathrm{a} 1}$ was $13.9 \%$ (compared to $E_{\mathrm{a} 4}=20.1 \%$ ). Deduction of Eq. (5) can be found in Supplementary Note 5 .

Estimation of solar radiance coverage enhancement. The effect of the LHCs on the performance of a bio-photoelectrode in response to visible light was estimated using

$$
\mathrm{U}_{\mathrm{LHCII}}=\frac{\int S(\lambda)\left(\mathrm{EQE}_{\mathrm{T}}(\lambda)-\mathrm{EQE}_{\mathrm{RC}}(\lambda)\right) \mathrm{d} \lambda}{\int S(\lambda) \mathrm{EQE}_{\mathrm{RC}}(\lambda) \mathrm{d} \lambda}
$$

where $S(\lambda)$ was the air mass 1.5 standard solar power reference spectrum as a function of wavelength (photons $\mathrm{m}^{-2} \mathrm{~nm}^{-1}$ ), $\mathrm{EQE}_{\mathrm{T}}$ was the $\mathrm{EQE}$ spectrum of each $\mathrm{LHC}+\mathrm{RC}$ system and $\mathrm{EQE}_{\mathrm{RC}}$ was that of the RC-only component (Supplementary Fig. 4, green versus magenta shade). Integration provided an estimate of the improvement in the use of solar energy $\left(U_{\mathrm{LHCII}}\right)$ between 400 and $700 \mathrm{~nm}$ where the chlorophyll-based LHCs absorb. Values are compiled in Table 1.

Protein structures and chimera modelling. Protein structures used in modelling were Protein Data Bank entries 3ZUW for the Rba. sphaeroides RC ${ }^{62}$, 2BHW for the LHCII from pea ${ }^{31}, 4 \mathrm{KX} 8$ for the LHCI from pea ${ }^{37}$ and 4MLI for SpyCatcher/ $\mathrm{Tag}^{42}$. Schematic models of chimeras were produced using Modeller ${ }^{63}$.

Reporting summary. Further information on research design is available in the Nature Research Reporting Summary linked to this article.

\section{Data availability}

The data underlying Figs. 1a-c, 2a-c, e, 3d-g and 4a-f, i as well as Supplementary Figs. 2, 4, 6-11, 18-20 are provided as a Source Data file. All data and biological materials reported in this study can be provided by the corresponding author upon reasonable request.

\section{Code availability}

Codes for data processing, analysis, plotting and simulation were implemented within MATLAB (release 2018b; MathWorks) and can be provided by the corresponding author on reasonable request.

Received: 8 January 2020; Accepted: 3 March 2020;

Published online: 24 March 2020

\section{References}

1. Kolber, Z. S. et al. Contribution of aerobic photoheterotrophic bacteria to the carbon cycle in the ocean. Science 292, 2492-2495 (2001).

2. Croce, R. \& van Amerongen, H. Natural strategies for photosynthetic light harvesting. Nat. Chem. Biol. 10, 492-501 (2014).

3. Saer, R. G. \& Blankenship, R. E. Light harvesting in phototrophic bacteria: structure and function. Biochem. J. 474, 2107-2131 (2017).

4. Scholes, G. D., Fleming, G. R., Olaya-Castro, A. \& van Grondelle, R. Lessons from nature about solar light harvesting. Nat. Chem. 3, 763-774 (2011).

5. Zinth, W. \& Wachtveitl, J. The first picoseconds in bacterial photosynthesisultrafast electron transfer for the efficient conversion of light energy. ChemPhysChem 6, 871-880 (2005).

6. Jones, M. R. The petite purple photosynthetic powerpack. Biochem. Soc. Trans. 37, 400-407 (2009)

7. Blankenship, R. E. et al. Comparing photosynthetic and photovoltaic efficiencies and recognizing the potential for improvement. Science 332, 805-809 (2011).

8. Ort, D. R. et al. Redesigning photosynthesis to sustainably meet global food and bioenergy demand. Proc. Natl Acad. Sci. USA 112, 8529-8536 (2015).

9. Wang, F., Liu, X. \& Willner, I. Integration of photoswitchable proteins, photosynthetic reaction centers and semiconductor/biomolecule hybrids with electrode supports for optobioelectronic applications. Adv. Mater. 25, 349-377 (2013).

10. Nguyen, K. \& Bruce, B. D. Growing green electricity: progress and strategies for use of Photosystem I for sustainable photovoltaic energy conversion. Biochim. Biophys. Acta 1837, 1553-1566 (2014).

11. Yehezkeli, O., Tel-Vered, R., Michaeli, D., Willner, I. \& Nechushtai, R. Photosynthetic reaction center-functionalized electrodes for photobioelectrochemical cells. Photosynth. Res. 120, 71-85 (2014).

12. Ravi, S. K. \& Tan, S. C. Progress and perspectives in exploiting photosynthetic biomolecules for solar energy harnessing. Energy Environ. Sci. 8, 2551-2573 (2015). 
13. Friebe, V. M. \& Frese, R. N. Photosynthetic reaction center-based biophotovoltaics. Curr. Opin. Electrochem. 5, 126-134 (2017).

14. Milano, F., Punzi, A., Ragni, R., Trotta, M. \& Farinola, G. M. Photonics and optoelectronics with bacteria: making materials from photosynthetic microorganisms. Adv. Funct. Mater. 1805521, 1-17 (2018).

15. Ravi, S. K. et al. Photosynthetic bioelectronic sensors for touch perception, UV-detection, and nanopower generation: toward self-powered e-skins. Adv. Mater. 1802290, 1-9 (2018).

16. Sokol, K. P. et al. Bias-free photoelectrochemical water splitting with Photosystem II on a dye-sensitized photoanode wired to hydrogenase. Nat. Energy 3, 944-951 (2018).

17. Tan, S. C., Crouch, L. I., Jones, M. R. \& Welland, M. Generation of alternating current in response to discontinuous illumination by photoelectrochemical cells based on photosynthetic proteins. Angew. Chem. Int. Ed. 51, 6667-6671 (2012).

18. Mirvakili, S. M. et al. Photoactive electrodes incorporating electrosprayed bacterial reaction centers. Adv. Funct. Mater. 24, 4789-4794 (2014).

19. Yaghoubi, H. et al. Large photocurrent response and external quantum efficiency in biophotoelectrochemical cells incorporating reaction center plus light harvesting complexes. Biomacromolecules 16, 1112-1118 (2015).

20. Friebe, V. M. et al. Plasmon-enhanced photocurrent of photosynthetic pigment proteins on nanoporous silver. Adv. Funct. Mater. 26, 285-292 (2016).

21. Ravi, S. K. et al. A mechanoresponsive phase-changing electrolyte enables fabrication of high-output solid-state photobioelectrochemical devices from pigment-protein multilayers. Adv. Mater. 30, 1-8 (2018).

22. Gundlach, K., Werwie, M., Wiegand, S. \& Paulsen, H. Filling the 'green gap' of the major light-harvesting chlorophyll a/b complex by covalent attachment of Rhodamine Red. Biochim. Biophys. Acta 1787, 1499-1504 (2009).

23. Dutta, P. K. et al. Reengineering the optical absorption cross-section of photosynthetic reaction centers. J. Am. Chem. Soc. 136, 4599-4604 (2014).

24. Yoneda, Y. et al. Extension of light-harvesting ability of photosynthetic lightharvesting complex 2 (LH2) through ultrafast energy transfer from covalently attached artificial chromophores. J. Am. Chem. Soc. 137, 13121-13129 (2015).

25. Werwie, M., Fehr, N., Xu, X., Basché, T. \& Paulsen, H. Comparison of quantum dot-binding protein tags: affinity determination by ultracentrifugation and FRET. Biochim. Biophys. Acta 1840, 1651-1656 (2014).

26. Nabiev, I. et al. Fluorescent quantum dots as artificial antennas for enhanced light harvesting and energy transfer to photosynthetic reaction centers. Angew. Chem. Int. Ed. 49, 7217-7221 (2010)

27. Liu, J., Mantell, J., Di Bartolo, N. \& Jones, M. R. Mechanisms of self-assembly and energy harvesting in tuneable conjugates of quantum dots and engineered photovoltaic proteins. Small 15, 1804267 (2019).

28. Wax, T. J. \& Zhao, J. Optical features of hybrid molecular/biological-quantum dot systems governed by energy transfer processes. J. Mater. Chem. C. 7, 6512-6526 (2019).

29. Grayson, K. J. et al. Augmenting light coverage for photosynthesis through YFP-enhanced charge separation at the Rhodobacter sphaeroides reaction centre. Nat. Commun. 8, 1-12 (2017).

30. Liu, Z. et al. Crystal structure of spinach major light-harvesting complex at 2.72 A resolution. Nature 428, 287-292 (2004)

31. Standfuss, J., Van Scheltinga, A. C. T., Lamborghini, M. \& Kühlbrandt, W. Mechanisms of photoprotection and nonphotochemical quenching in pea light-harvesting complex at $2.5 \AA$ resolution. EMBO J. 24, 919-928 (2005).

32. Su, X., Wei, X., Zhu, D., Chang, W. \& Liu, Z. Structure and assembly mechanism of plant $\mathrm{C}_{2} \mathrm{~S}_{2} \mathrm{M}_{2}$-type PSII-LHCII supercomplex. Science 820, 815-820 (2017).

33. Van Bezouwen, L. S. et al. Subunit and chlorophyll organization of the plant Photosystem II supercomplex. Nat. Plants 3, 1-11 (2017).

34. Croce, R., Morosinotto, T., Castelletti, S., Breton, J. \& Bassi, R. The Lhca antenna complexes of higher plants Photosystem I. Biochim. Biophys. Acta Bioenerg. 1556, 29-40 (2002).

35. Ben-Shem, A., Frolow, F. \& Nelson, N. Crystal structure of plant Photosystem I. Nature 426, 630-635 (2003).

36. Wientjes, E. \& Croce, R. The light-harvesting complexes of higher-plant Photosystem I: Lhca1/4 and Lhca2/3 form two red-emitting heterodimers. Biochem. J. 433, 477-485 (2011).

37. Qin, X., Suga, M., Kuang, T. \& Shen, J.-R. Structural basis for energy transfer pathways in the plant PSI-LHCI supercomplex. Science 348, 989-995 (2015).

38. Krüger, T. P. J., Wientjes, E., Croce, R. \& van Grondelle, R. Conformational switching explains the intrinsic multifunctionality of plant light-harvesting complexes. Proc. Natl Acad. Sci. USA 108, 13516-13521 (2011).

39. Friebe, V. M. et al. On the mechanism of ubiquinone mediated photocurrent generation by a reaction center based photocathode. Biochim. Biophys. Acta Bioenerg. 1857, 1925-1934 (2016).

40. Friebe, V. M., Millo, D., Swainsbury, D., Jones, M. R. \& Frese, R. N. Cytochrome $\mathrm{c}$ provides an electron-funneling antenna for efficient photocurrent generation in a reaction center biophotocathode. ACS Appl. Mater. Interfaces 9, 23379-23388 (2017).
41. Zakeri, B. et al. Peptide tag forming a rapid covalent bond to a protein, through engineering a bacterial adhesin. Proc. Natl Acad. Sci. USA 109, 690-697 (2012)

42. Li, L., Fierer, J. O., Rapoport, T. A. \& Howarth, M. Structural analysis and optimization of the covalent association between SpyCatcher and a peptide tag. J. Mol. Biol. 426, 309-317 (2014).

43. Natali, A., Roy, L. M. \& Croce, R. In vitro reconstitution of light-harvesting complexes of plants and green algae. J. Vis. Exp. 92, 1-13 (2014).

44. Hobe, S., Prytulla, S., Kuhlbrandt, W. \& Paulsen, H. Trimerization and crystallization of reconstituted light-harvesting chlorophyll a/b complex. EMBO J. 13, 3423-3429 (1994).

45. Caffarri, S., Croce, R., Cattivelli, L. \& Bassi, R. A look within LHCII Differential analysis of the Lhcb1-3 complexes building the major trimeric antenna complex of higher-plant photosynthesis. Biochemistry 43, 9467-9476 (2004).

46. Croce, R., Weiss, S. \& Bassi, R. Carotenoid-binding sites of the major lightharvesting complex II of higher plants. J. Biol. Chem. 274, 29613-29623 (1999).

47. Schmid, V. H., Cammarata, K. V., Bruns, B. U. \& Schmidt, G. W. In vitro reconstitution of the photosystem I light-harvesting complex LHCI-730: heterodimerization is required for antenna pigment organization. Proc. Natl Acad. Sci. USA 94, 7667-7672 (1997).

48. Passarini, F., Wientjes, E., van Amerongen, H. \& Croce, R. Photosystem I light-harvesting complex Lhca4 adopts multiple conformations: Red forms and excited-state quenching are mutually exclusive. Biochim. Biophys. Acta Bioenerg. 1797, 501-508 (2010).

49. Hauska, G., Schoedl, T., Remigy, H. \& Tsiotis, G. The reaction center of green sulfur bacteria. Biochim. Biophys. Acta Bioenergetics 1507, 260-277 (2001).

50. Gisriel, C. et al. Structure of a symmetric photosynthetic reaction centerphotosystem. Science 357, 1021-1025 (2017).

51. Ihalainen, J. A. et al. Excitation decay pathways of Lhca proteins: A timeresolved fluorescence study. J. Phys. Chem. B 109, 21150-21158 (2005).

52. Veggiani, G. et al. Programmable polyproteams built using twin peptide superglues. Proc. Natl Acad. Sci. USA 113, 1202-1207 (2016).

53. Buldun, C. M., Jean, J. X., Bedford, M. R. \& Howarth, M. SnoopLigase catalyzes peptide-peptide locking and enables solid-phase conjugate isolation. J. Am. Chem. Soc. 140, 3008-3018 (2018).

54. Swainsbury, D. J. K., Friebe, V. M., Frese, R. N. \& Jones, M. R. Evaluation of a biohybrid photoelectrochemical cell employing the purple bacterial reaction centre as a biosensor for herbicides. Biosens. Bioelectron. 58, 172-178 (2014)

55. Jones, M. R. et al. Mutants of Rhodobacter sphaeroides lacking one or more pigment-protein complexes and complementation with reaction-centre, LH1 and LH2 genes. Mol. Microbiol. 6, 1173-1184 (1992).

56. Lichtenthaler, H. \& Wellburn, A. Determinations of total carotenoids and chlorophylls b of leaf extracts in different solvents. Biochem. Soc. Trans. 11, 591-592 (1983).

57. Ma, J. \& Xia, D. The use of blue native PAGE in the evaluation of membrane protein aggregation states for crystallization. J. Appl. Crystallogr. 41, 1150-1160 (2008).

58. Gardecki, J. A. \& Maroncelli, M. Set of secondary emission standards for calibration of the spectral responsivity in emission spectroscopy. Appl. Spectrosc. 52, 1179-1189 (1998).

59. Würth, C., Grabolle, M., Pauli, J., Spieles, M. \& Resch-Genger, U. Relative and absolute determination of fluorescence quantum yields of transparent samples. Nat. Protoc. 8, 1535-1350 (2013).

60. Rurack, K. \& Spieles, M. Fluorescence quantum yields of a series of red and near-infrared dyes emitting at 600-1000 nm. Anal. Chem. 83, 1232-1242 (2011).

61. Krüger, T. P. J., Novoderezhkin, V. I., Ilioaia, C. \& van Grondelle, R. Fluorescence spectral dynamics of single LHCII trimers. Biophys. J. 98, 3093-3101 (2010).

62. Gibasiewicz, K. et al. Mechanism of recombination of the $\mathrm{P}^{+} \mathrm{H}_{\mathrm{A}}{ }^{-}$radical pair in mutant Rhodobacter sphaeroides reaction centers with modified free energy gaps between $\mathrm{P}^{+} \mathrm{B}_{\mathrm{A}}{ }^{-}$and $\mathrm{P}^{+} \mathrm{H}_{\mathrm{A}}{ }^{-}$. J. Phys. Chem. B 115, 13037-13050 (2011).

63. Webb, B. \& Sali, A. Comparative protein structure modeling using MODELLER. Curr. Protoc. Bioinformatics 15, 5.6.1-5.6.30 (2016).

\section{Acknowledgements}

The Lhcb1.3 plasmid was a kind gift from Prof. Roberta Croce of the Vrije Universitei Amsterdam, The Netherlands. We also thank Dr. Majid Mosayebi from the School of Mathematics, University of Bristol for generous advice on simulations. J.L. and M.R.J. acknowledge funding from the EPSRC/BBSRC Synthetic Biology Centre for Doctoral Training (EP/L016494/1) and from the BrisSynBio Synthetic Biology Research Centre at the University of Bristol (BB/L01386X/1). R.N.F. acknowledges support from the Netherlands Organisation for Scientific Research (NWO) for a Vidi grant and V.M.F. for funding from NWO Veni project 16866. 


\section{Author contributions}

J.L. and M.R.J. conceived the research. J.L. engineered the chimeras, characterised binding and energy transfer and conducted the simulations. V.M.F. and J.L. carried out photochronoamperometry under supervision from R.N.F. J.L. and M.R.J. drafted the manuscript and all authors commented on the manuscript. M.R.J. supervised the project.

\section{Competing interests}

The authors declare no competing interests.

\section{Additional information}

Supplementary information is available for this paper at https://doi.org/10.1038/s41467020-15321-w.

Correspondence and requests for materials should be addressed to M.R.J.

Peer review information Nature Communications thanks Thomas Beatty and the other, anonymous, reviewer(s) for their contribution to the peer review of this work. Peer reviewer reports are available.
Reprints and permission information is available at http://www.nature.com/reprints

Publisher's note Springer Nature remains neutral with regard to jurisdictional claims in published maps and institutional affiliations.

(c) Open Access This article is licensed under a Creative Commons Attribution 4.0 International License, which permits use, sharing, adaptation, distribution and reproduction in any medium or format, as long as you give appropriate credit to the original author(s) and the source, provide a link to the Creative Commons license, and indicate if changes were made. The images or other third party material in this article are included in the article's Creative Commons license, unless indicated otherwise in a credit line to the material. If material is not included in the article's Creative Commons license and your intended use is not permitted by statutory regulation or exceeds the permitted use, you will need to obtain permission directly from the copyright holder. To view a copy of this license, visit http://creativecommons.org/ licenses/by/4.0/.

(C) The Author(s) 2020 